\title{
Thiodicarbe : distribution tissulaire post-mortem suite à une intoxication volontaire
}

\section{Thiodicarb: Post-mortem tissue distribution following a self-poisoning}

\author{
Guillaume HOIZEY ${ }^{(1,2) *}$, Frédéric CANAS ${ }^{(2)}$, Laurent BINET ${ }^{(1)}$, \\ Gérard JEUNEHOMME ${ }^{(2)}$, Marie-Hélène BERNARD(2), \\ Christian BOULANGER ${ }^{(1)}$, Denis LAMIABLE ${ }^{(1,2)}$
}

(1) Laboratoire de Pharmacologie et Toxicologie, Hôpital Maison-Blanche, CHU de Reims, France (2) Fédération Médico-Judiciaire de CHU de Reims, France

*Auteur à qui adresser la correspondance : Guillaume HOIZEY, Laboratoire de Pharmacologie et de Toxicologie, CHU de Reims, 45, rue Cognacq-Jay, F-51092 Reims Cedex, France Tél : (33) 326787530 - Fax : (33) 326788456 -E-mail : ghoizey@chu-reims.fr

(Reçu le 11 octobre 2006 ; accepté après modification le 19 mars 2007)

\section{RÉSUMÉ}

Le thiodicarbe est un insecticide liquide de la famille des carbamates anticholinestérasiques, dont l'activité est en partie liée à son principal produit de dégradation, le méthomyl. Une femme de 40 ans est retrouvée sans vie dans son véhicule au côté de plusieurs emballages de médicaments et. d'un flacon ouvert de Larvin@ (thiodicarbe). L'autopsie ne permet pas de déterminer l'origine de la mort, et laisse suspecter; du fait des circonstances, une origine toxique dans un but d'autolyse. Le contenu gastrique ainsi que les prélèvements sanguins et tissulaires effectués lors de l'examen postmortem sont adressés au laboratoire pour analy'ses toxicologiques. Ces analyses ont permis d'identifier dans le sang, les urines et le contenu gastrique de la victime, plusieurs substances médicamenteuses, en particulier; le zolpidem, le bromazépam, le nordiazépam et la lévoprémazine dont les concentrations sanguines ont été retrouvées nettement supérieures aux valeurs connues pour être thérapeutiques. Les dosages spécifiques du thiodicarbe et du méthomyl ont été réalisés par CLHP-SM/SM à trappe d'ions dans l'ensemble

\section{SUMMARY}

Thiodicarb is a non-systemic carbanate insecticide whose acetylcholinesterase activity is related to its main methomy? degradation product. A 40-year-old woman was found dead in her car: Empty packages of medicines and an open bottle of Lavin $@$ containing thiodicarb were found near her body. No signs of violence nor traumatic injuries were noticed upon autopsy, and police investigations strongly suggested a suicide. Post-mortem peripheral blood, gastric content, urine, bile, vitreous humor; liver; kidney, lung, heart, and brain samples were submitted for toxicological analysis. Systematic analysis revealed the presence of various sedatives, hypnotics and antipsychotic drugs in blood, urine and gastric content. Some of the compounds identified were determined at blood concentrations well above the known therapeutic concentrations. Specific analysis of thiodicarb and of its methomyl metabolite was then performed on all. fluids and tissues collected during autopsy by liquid-chromatography ion-trap tandem mass spectronetry (LC-MS$M S)$. 
des prélèvements autopsiques. Le pouvoir anticholinestérasique du sang, des urines et du contenu gastrique était respectivement de 83,82 et $32 \%$ (normale : 0\%). L'analyse du contenu de la bouteille découverte sur la scène, confirmait la nature du composé. Alors que le thiodicarbe n'a été mis en évidence que dans le contenu gastrique, son principal métabolite a été détecté dans la plupart des tissus et fluides autopsiques analysés. Ceci peut s'expliquer par l'instabilité du thiodicarbe dans un environnement acide, tel que l'estomac. Les concentrations de méthonyl mesurées dans la plupart des tissus et/ou fluides sont compatibles avec les concentrations mesurées dans des cas similaires, bien que ces dernières soient sujettes à une importante variabilité.

La forte inhibition cholinestérasique est vraisemblablement à l'origine d'une paralysie respiratoire et de troubles hémodynamiques qui, conjugués à la toxicité des substances médicamenteuses également présentes en quantités importantes, ont conduit à une dépression respiratoire majeure fatale en l'absence de prise en charge thérapeutique rapide.

\section{Introduction}

Le thiodicarbe (CAS N59669-26-0) est un insecticide liquide de la famille des carbamates inhibiteurs des cholinestérases. Commercialisé en France sous le nom de Larvin ${ }^{\circledR}$, le thiodicarbe était, avant son retrait du marché en 2003, fréquemment utilisé dans les régions viticoles pour le traitement des parcelles de vignes, notamment dans la lutte contre les tordeuses de la grappe et la pyrale $(1,2)$.

Sur le plan de sa structure chimique, le thiodicarbe est composé de deux molécules de méthomyl (CAS $n^{\circ} 16752-77-5$ ), autre carbamate anticholinestérasique, reliées au niveau de leur groupement amine par un atome de soufre (Figure 1).

Après ingestion par voie orale, le thiodicarbe est rapidement dégradé en méthomyl et en divers autres composés intermédiaires instables tels que le méthomyl oxime, le méthomyl méthylol, le méthomyl sulfoxyde ou le méthomyl sulfoxyde oxime (3).

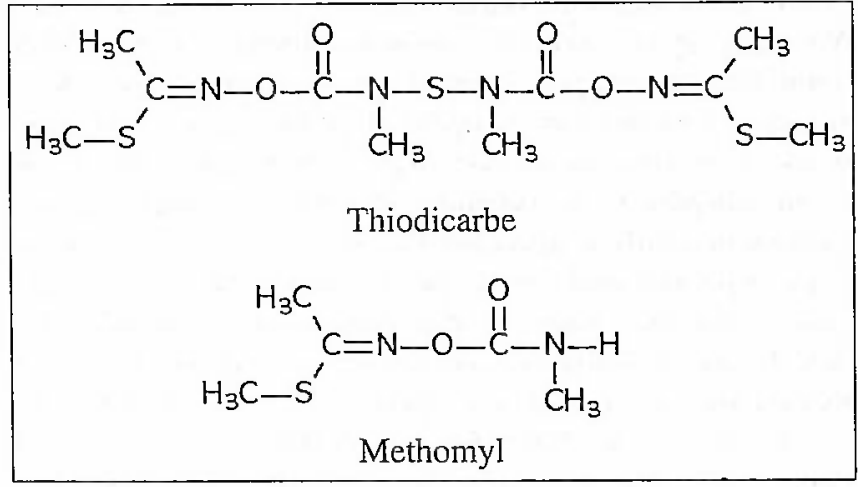

Figure 1 : Structures chimiques du thiodicarbe et du méthomyl.
The anticholinesterase capacity of blood, urine and gastric content collected at autopsy were 83,82 , and $32 \%$, respectively (normal value: $0 \%$ ). The presence of thiodicarb in the bottle found near the body confirms the hypothesis of an intake of that compound. Although thiodicarb was only detected in gastric content, its methomyl metabolite was quantified in most post-mortem tissues and fuids. This is probably related to the poor stability of thiodicarb in an acidic environment such as the stomach. Concentrations of methomyl in tissues and fluids were consistent with those measured in similar fatal cases, in spite of a large variability in published results. Methomyl was found to distribute in almost all tissues and fluids investigated.

It is thus suggested that the paralysis of respiratory muscles, likely induced by methomyl, in addition to the toxic concentrations of respiratory depressant drugs such benzodiazepines, could have facilitated death.

L'intoxication par une surdose d'un pesticide de type carbamate anticholinestérasique provoque généralement des manifestations proches de celles d'une intoxication organophosphoré avec classiquement, des signes digestifs précoces, des signes d'intoxication muscarinique avec bradycardie, hypotension artérielle et dyspnée asthmatiforme, des signes d'intoxication nicotinique avec coma convulsif, risque de choc hémodynamique et paralysie (en particulier des muscles respiratoires) (4). Le décès est souvent la conséquence de l'aśphyxie provoquée par cette paralysie.

A notre connaissance, la littérature ne rapporte aucun décès résultant d'une exposition au thiodicarbe. Nous décrivons le cas d'un décès faisant suite à l'absorption volontaire de thiodicarbe et d'autres substances médicamenteuses, avec détermination des concentrations tissulaires post-mortem de thiodicarbe et de son principal produit de dégradation, le méthomyl. Le dosage spécifique de ces deux composés dans les différents fluides et tissus prélevés lors de l'autopsie a été réalisé par chromatographie en phase liquide couplée à la spectrométrie de masse à trappe d'ions.

\section{Cas médico-légal}

Une femme de 40 ans est retrouvée sans vie dans son véhicule, avec à ses côtés, plusieurs emballages de médicaments vides et un flacon ouvert, étiqueté au nom de LARVIN@. L'autopsie pratiquée environ trois jours après la découverte du corps ne permet pas de déterminer l'origine de la mort. En particulier, il n'y pas d'élément évocateur de traumatismes ou d'une mort violente. Du fait des circonstances particulières entourant ce décès, une origine toxique à visée suicidaire est envisagée. 
Les prélèvements autopsiques suivants nous sont adressés pour analyses toxicologiques : contenu gastrique, sang cardiaque et périphérique, urine, bile, humeur vitrée, foie, rein, poumon, cerveau et cœur. Les échantillons ont été conservés congelés à $-20^{\circ} \mathrm{C}$ jusqu'à analyse.

\section{Matériels et Méthodes}

\section{Analyses toxicologiques}

La recherche des alcools (éthanol, méthanol, acétone et isopropanol) a été effectuée dans le sang périphérique par chromatographie en phase gazeuse avec détection par ionisation de flamme (CPG-FID).

La recherche des stupéfiants (opiacés, cannabinoïdes, cocaïnes et amphétamines) a été réalisée par méthode immunoenzymatique dans les urines et complétée par une recherche dans le sang périphérique par $\mathrm{CPG}$ couplée à la spectrométrie de masse (SM).

Une recherche élargie a été effectuée sur le sang périphérique, les urines et le contenu gastrique par CPGSM et complétée par une recherche à large spectre au moyen de la chromatographie en phase liquide couplée à la SM en tandem à trappe d'ions (CLHP-SM/SM). Pour cette dernière, l'usage d'une trappe d'ions permet l'emploi d'un mode SM/SM particulier : l'exclusion dynamique d'ions. Ce mode associé à des conditions chromatographiques spécifiques (gradient sur 20 minutes) autorisent l'identification d'un grand nombre de substances médicamenteuses ou non, via l'utilisation d'une bibliothèque spectrale construite en mode SM/SM et contenant, à ce jour, un peu plus de 500 spectres. Les échantillons biologiques ont été analysés après extraction liquide-liquide en milieu alcalin et acide. En cas de recherche positive, la quantification des molécules identifiées était réalisée par CLHPSM/SM à trappe d'ions.

Du fait du contexte dans lequel le cadavre a été découvert (présence à ses côtés d'un flacon étiqueté Larvin $(\mathbb{R})$, une recherche spécifique du thiodicarbe et du méthomyl a été effectué sur l'ensemble des prélèvements autopsiques.

\section{Dosage spécifique du thiodicarbe et du méthomyl}

\section{Réactifs}

Le thiodicarbe, le méthomyl et le carisoprodol ont été achetés chez SIGMA (France). L'ensemble des solvants organiques et réactifs utilisés sont de qualité analytique. L'acétonitrile et le dichlorométhane proviennent de la société SDS (Peypin, France); le méthanol et l'acide formique ont été obtenus chez Merck
(Darmstadt, Allemagne). L'eau purifiée est produite par un système Waters-MilliQ® purification system (Millipore, Saint-Quentin en Yvelines, France).

\section{Extraction des échantillons}

Les échantillons sanguins, d'urine, de contenu gastrique $(100 \mu \mathrm{L})$ et les extraits tissulaires ( $100 \mu \mathrm{L}$ d'un broyat obtenu à partir d'environ $100 \mathrm{mg}$ de tissu frais, placés dans $1 \mathrm{~mL}$ d'eau purifiée) sont extraits en milieu basique par $2 \mathrm{~mL}$ de dichlorométhane après ajout de $10 \mu \mathrm{L}$ d'une solution de carisoprodol $[200 \mathrm{mg} / \mathrm{L}$ dans le méthanol], utilisé comme étalon interne, et $100 \mu \mathrm{L}$ de carbonate de sodium à $20 \%$. Après agitation au vortex durant $1 \mathrm{~min}$ et centrifugation à $3000 \mathrm{~g}$ pendant $5 \mathrm{~min}$, la phase organique est transférée dans un tube conique puis évaporée à sec sous un courant d'azote à $40^{\circ} \mathrm{C}$. L'extrait sec est repris par $200 \mu \mathrm{L}$ du mélange acide formique $0,1 \% /$ acétonitrile $(50 / 50 \mathrm{v} / \mathrm{v})$, et $20 \mu \mathrm{L}$ d'échantillon sont injectés dans le système chromatographique.

\section{Instrumentation et conditions d'analyse}

Le système utilisé est constitué d'un chromatographe en phase liquide Surveyor ${ }^{\circledR}$ LC system couplé à un détecteur de masse à trappe d'ions LCQ Advantage $($ (ThermoElectron, Les Ulis, France). L'ensemble est piloté par le logiciel dédié Xcalibur(B). La séparation chromatographique des analytes est effectuée au moyen d'une colonne Hypurity ${ }^{\circledR}$ C18 (150 x 2,1 mm d.i. ; 5- $\mu \mathrm{m}$; ThermoHypersil-Keystone, Les Ulis, France) maintenue à une température de $30^{\circ} \mathrm{C}$. La phase mobile, constituée du mélange acide formique $0,1 \% /$ acétonitrile $(50 / 50 \mathrm{v} / \mathrm{v})$, est délivrée à un débit de $0,3 \mathrm{~mL} / \mathrm{min}$ en mode isocratique. L'interface d'ionisation est de type électrospray (ESI) utilisée en mode positif. Les principaux paramètres du détecteur sont : tension entre l'aiguille de nébulisation et le corps de la source : $4 \mathrm{kV}$; température de source et de désolvatation de l'azote : respectivement $200^{\circ} \mathrm{C}$ et $300^{\circ} \mathrm{C}$; tension de l'électromultiplicateur : $400 \mathrm{~V}$. L'hélium ultrapur $(99,995 \%)$ est introduit dans la trappe en tant que gaz de collision (pression : $5.10^{-3}$ Torr). Les données sont enregistrées en mode SM/SM et acquisition de spectres complets (Mode «Full scan $M S / M S »)$ avec un balayage de $m / z 120$ à $m / z 600$. Les spectres SM/SM sont obtenus après collision de l'ion parent (ion pseudomoléculaire protoné $[\mathrm{M}+\mathrm{H}]^{+}$) par l'hélium [énergie de collision : 50\% (unité arbitraire)]. Les ions parents et fils (correspondant au spectre « complet »SM/SM), et les temps de rétention sont présentés dans le tableau I.

Dans ces conditions, la limite de quantification pour le thiodicarbe a pu être déterminée à $0,5 \mathrm{mg} / \mathrm{L}$ dans le sang total, et celle du méthomyl à $0,1 \mathrm{mg} / \mathrm{L}$. Les courbes de calibrations ( 5 points de gamme) étaient 
Tableau I : Temps de rétention (Tr) et fragments ioniques (ions parents et fils) du thiodicarbe, du méthomyl et du carisoprodol, utilisé comme étalon interne. L'ion fils souligné est employé pour la quantification.

\begin{tabular}{|c|c|c|c|}
\hline Molécule & $\operatorname{Tr}(\min )$ & Ion parent $(\mathrm{m} / \mathrm{z})$ & Ions fils $(\mathrm{m} / \mathrm{z})^{*}$ - Spectre complet SM/SM \\
\hline Thiodicarbe & 2,31 & $355(\mathrm{M}+\mathrm{H})^{+}$ & $\underline{163(100), 209(26), 193(17)}$ \\
\hline Méthomyl & 1,76 & $163(\mathrm{M}+\mathrm{H})^{+}$ & $\underline{122(100), 88(63)}$ \\
\hline Carisoprodol & 2,66 & $261(\mathrm{M}+\mathrm{H})^{+}$ & $\underline{176}(100), 158(22), 200(11)$ \\
\hline
\end{tabular}

* Les valeurs entre parenthèses correspondent aux intensités relatives.

linéaires jusqu'à la concentration de $50 \mathrm{mg} / \mathrm{L}$ pour le thiodicarbe et de $20 \mathrm{mg} / \mathrm{L}$ pour le méthomyl. Bien que la validation complète de la méthode de dosage du thiodicarbe et du méthomyl n'ait pas été réalisée selon les critères habituels, une simple mesure de la reproductibilité intra-jour à partir d'échantillons sanguins $(n=6)$ surchargés à une concentration de $5 \mathrm{mg} / \mathrm{L}$ de thiodicarbe et de méthomyl, montrait des coefficients de variations inférieurs à $10 \%$.

\section{Détermination de la capacité anticholi- nestérasique}

Le pouvoir anticholinestérasique du sérum, des urines et du contenu gastrique a également été recherché, selon une méthode adaptée de Mahieu P. et coll. (5). A cet effet, l'activité cholinestérasique (dosage des cholinestérases) des trois matrices biologiques suscitées a été déterminée au moyen du réactif Flex ${ }^{\circledR}$ Pseudocholinestérase (Dade Behring, France). Parallèlement, l'activité cholinestérasique d'un sérum témoin a été mesurée. Dans un second temps, cette activité a été déterminée, pour chaque matrice, sur le mélange constitué de $100 \mu \mathrm{L}$ de sérum témoin $(\mathrm{T})$ et de $100 \mu \mathrm{L}$ de sérum, d'urine ou de contenu gastrique (P), après incubation de l'ensemble pendant 15 minutes à $37^{\circ} \mathrm{C}$. La capacité anticholinestérasique, exprimée en pourcentage, est déterminée selon la formule suivante :

Capacité anticholinesiérasique $=100 \times\left[1-\frac{2 \times(\text { activité du mélange } T+P)}{\text { activité }(T)+\operatorname{activité~}(P)}\right]$

En l'absence de composés anticholinestérasiques dans les matrices biologiques testées, cet index doit approcher la valeur de $0 \%$.

\section{Résultats et discussion}

La recherche d'alcools montre une éthanolémie très faible de $0,11 \mathrm{~g} / \mathrm{L}$. La recherche de stupéfiants dans le sang périphérique et les urines est négative.

La recherche à large spectre pratiquée sur le sang périphérique, l'urine et le contenu gastrique, révèle la présence de nombreuses molécules psychotropes dont certaines ont été retrouvées à des concentrations sanguines nettement supérieures aux concentrations connues pour être thérapeutiques (tableau II).

Il convient de souligner que cette recherche élargie n'a pas permis, dans nos conditions analytiques, d'identifier lors des toutes premières analyses, le thiodicarbe ou le méthomyl. Ce fait s'explique principalement par la non disponibilité de ces molécules dans notre bibliothèque spectrale construite en CLHP-SM/SM à trappe d'ions (environ 500 spectres). Toutefois, le ré-examen des chromatogrammes plusieurs jours après les analyses initiales, montrait la présence de pics inconnus qui, une fois les composés recherchés disponibles, correspondaient clairement au thiodicarbe et à son métabolite.

Par ailleurs, l'instabilité du thiodicarbe et du méthomyl à températures élevées rend illusoire toute tentative de détection par notre méthode de recherche élargie au moyen de la CPG-SM $(6,7)$. Les rares méthodes présentées dans des cas similaires ont recourt le plus souvent à une dérivation des molécules $(6,8,9)$, parfois même après hydrolyse du méthomyl en son dérivé oxime (10). Ce sont finalement les éléments de l'enquête policière qui ont permis de nous orienter vers une possible ingestion de thiodicarbe.

L'analyse du contenu du flacon étiqueté Larvin® et retrouvé près du corps de la victime, a permis de confirmer la nature du produit ingéré.

Tableau II : Concentrations sanguines des substances médicamenteuses identifiées dans le liquide gastrique, les urines et le sang de la victime.

\begin{tabular}{|c|c|c|}
\hline $\begin{array}{c}\text { Composé } \\
\text { identifié }\end{array}$ & $\begin{array}{c}\text { Concentration } \\
\text { sanguine } \\
\text { (mg/L) }\end{array}$ & $\begin{array}{c}\text { Concentrations } \\
\text { thérapeutiques } \\
\text { (mg/L) }\end{array}$ \\
\hline Zolpidem & 2,87 & $0,08-0,15(0,20)$ \\
\hline Lormétazépam & 0,06 & $0,001-0,02$ \\
\hline Nordiazépam & 4,21 & $0,2-0,8(1,8)$ \\
\hline Tétrazépam & 0,35 & $0,05-0,60(1,0)$ \\
\hline Bromazépam & 2,39 & $0,08-0,17$ \\
\hline Lévoprémazine & 0,64 & $0,02-0,15$ \\
\hline
\end{tabular}

* Données extrailes de "TIAFT reference blood list of therapestic and toxic substances" Document consultable à l'adresse suivante : http://www.tiaft.org/tmembers/tvidx.html 
Par ailleurs, la détermination de la capacité anticholinestérasique du contenu gastrique, du sang et de l'urine a révélé une inhibition très importante des cholinestérases d'un sérum témoin (83, 82 et $32 \%$ d'inhibition, respectivement pour le liquide gastrique, le sang et l'urine), objectivant ainsi la présence d'un composé au fort pouvoir anticholinestérasique dans les matrices étudiées.

Les concentrations de thiodicarbe et de méthomyl déterminées dans les différents fluides et tissus autopsiques sont présentées dans le tableau III. Une estimation de la quantité de thiođicarbe ingéré n'a pas été possible du fait de la non mesure à l'autopsie, du volume total du contenu gastrique.

De part son importante instabilité en milieu acide, le thiodicarbe se dégrade rapidement dans l'estomac en méthomyl et en divers autres composés dérivés (3), expliquant probablement pourquoi, en dehors du contenu gastrique, nous n'avons pas retrouvé de thiodicarbe dans les différents prélèvements analysés. Le méthomyl a pu être identifié et quantifié dans la plupart des tissus autopsiques. Les concentrations sanguines et tissulaires sont compatibles avec celles décrites dans de rares cas similaires, bien que les concentrations rapportées soient particulièrement disparates. A titre d'illustration, dans plusieurs cas d'intoxications mortelles imputées à l'ingestion de méthomyl, les concentrations mesurées dans le sang périphérique et dans le foie étaient retrouvées à des concentrations variant respectivement de 0,003 à $63,5 \mathrm{mg} / \mathrm{L}$ et de non détectable à $1,2 \mathrm{mg} / \mathrm{kg}(8,9,11)$.

Sur le plan de la distribution tissulaire, les concentrations de méthomyl mesurées dans la plupart des tissus sont plus élevées que dans le sang périphérique, suggérant ainsi une distribution ante-mortem importante du méthomyl, compatible avec le caractère lipophile de cette molécule (12). Contrairement aux cas similaires présentés par Moriya F. et coll., le méthomyl n'a pas été identifié dans l'humeur vitrée, alors que ce milieu est décrit par les auteurs comme une matrice de choix pour la mise en évidence de cette molécule $(8,11)$.

A l'évidence, l'interprétation de ces données reste délicate en raison : 1.) de la rareté des cas rapportés dans la littérature ; 2.) de l'absence d'information sur le délai exact entre l'ingestion et le décès ; 3 .) de l'instabilité des composés soufrés tel que le méthomyl dans les prélèvements post-mortem, $\mathrm{y}$ compris lorsqu'ils sont congelés $(8,13) ; 4$.) de l'existence possible d'un métabolisme post-mortem du méthomyl via l'action des estérases sanguines et tissulaires (8).

Les cas d'intoxications mortels faisant suite à l'ingestion de thiodicarbe et/ou de méthomyl sont très peu
Tableau III : Concentrations de thiodicarbe et de méthomyl déterminées dans les fuides et tissus biologiques prélevés lors de l'autopsie du corps de la victime.

\begin{tabular}{|c|c|c|}
\hline Fluides ou Tissus & $\begin{array}{c}\text { Thiodicarbe } \\
(\mathbf{m g} / \mathbf{L}) \text { ou }(\mathbf{m g} / \mathbf{k g})\end{array}$ & $\begin{array}{c}\text { Méthomyl } \\
(\mathbf{m g} / \mathrm{L}) \text { ou }(\mathbf{m g} / \mathbf{k g})\end{array}$ \\
\hline Contenu gastrique & 24,3 & 19,9 \\
\hline Sang périphérique & $<\mathrm{LQ}$ & 0,7 \\
\hline Urine & $<\mathrm{LQ}$ & 8,5 \\
\hline Bile & $<\mathrm{LQ}$ & 2,7 \\
\hline Humeur vitrée & $<\mathrm{LQ}$ & $<\mathrm{LQ}$ \\
\hline Foie & $<\mathrm{LQ}$ & 0,7 \\
\hline Rein & $<\mathrm{LQ}$ & 1,7 \\
\hline Poumon & $<\mathrm{LQ}$ & 1,5 \\
\hline Cerveau & $<\mathrm{LQ}$ & 9,3 \\
\hline Coeur & $<\mathrm{LQ}$ & 3,6 \\
\hline
\end{tabular}

LQ : Limite de quantification

décrits, particulièrement en France (aucun cas rapporté pour le thiodicarbe).

Dans cette affaire, les investigations policières et judiciaires ont conclus à une autolyse. Les analyses toxicologiques ne permettent pas d'imputer le décès à l'absorption exclusive de Larvin(B). Toutefois, il est vraisemblable que l'importante inhibition cholinestérasique liée à la présence de méthomyl dans l'organisme ait été à l'origine d'une paralysie respiratoire et de troubles hémodynamiques sévères, potentiellement majorés par la présence, à des concentrations toxiques, de substances médicamenteuses, dont certaines présentent la particularité d'être dépresseurs respiratoires. L'action conjuguée de ces molécules a pu conduire à un coma et à une dépression respiratoire fatale en l'absence de prise en charge thérapeutique rapide.

\section{Conclusion}

Cette intoxication volontaire mortelle impliquant le thiodicarbe est probablement une des seules rapportées à ce jour. Il convient, toutefois, de souligner que ce sont les informations recueillies au moment de la levée de corps qui nous ont permis d'orienter nos investigations toxicologiques vers ce puissant carbamate anticholinestérasique. L'étude de la distribution tissulaire a permis de confirmer l'imprégnation d'une grande partie de l'organisme par le méthomyl après ingestion de thiodicarbe. Enfin, ce cas met en lumière la difficulté de faire procéder à la destruction effective des produits phytosanitaires potentiellement dangereux dont le retrait du marché a été décidé il y a déjà plusieurs années. 


\section{Références}

1. Plan de surveillance résidus en Viticulture (campagnes viticole 1990-2003) - Direction Générale de l'Alimentation - Sous direction de la Qualité et de la protection des Végétaux. Ce document peut être consulté sur le site :

http://www.avignon.inra.fr/stcavignon/centre/unites/grappa/pdf/EnqueteRaisinVin9003.pdf

2. Ce document peut être consulté sur le site : http://e-phy.agriculture.gouv.fr/spe/ppnull.htm

3. Ce document peut être consulté sur le site : http://www.inchem.org/documents/jmpr/jmpmono/v00pr0 9.htm

4. Bismuth C., Baud F., Conso F., Dally S., Fréjaville J .P., Garnier R., Jaeger A. Toxicologie Clinique $5^{\text {enie }}$ édition. Médecine-Sciences, Flammarion, 2000 : 516-24.

5. Mahieu P., Hassoun A., Van Binst R., Lauwerys R., Deheneffe Y. Severe and prolonged poisoning by fenthion. Significance of the determination of the anticholinesterase capacity of plasma. J. Toxicol. Clin. Toxicol. 1982 ; 19(5): 425-32.

6. Miyazaki T., Yashiki M., Kojima T., Chikasue F., Ochiai A., Hidani Y. Fatal and non-fatal methomyl intoxication in an attempted double suicide. Forensic Sci. Int. $1989 ; 42$ : 263-70.

7. Alawadi A.H., Wasfi A., Alqawas A.S., Zorob O.M. Five fatal cases due to methomyl poisoning. Bulletin of The International Association of Forensic Toxicologists. $2001 ; 31(1): 6-8$.

8. Moriya F., Hashimoto Y. A fatal poisoning by methomyl and nicotine. Forensic Sci. Int. 2005 ; 149 : 167-70.

9. Tsatsakis A.M., Tsakalof A.K., Michalodimitrakis E.N. The analysis of methomyl, a carbamate pesticide, in postmortem samples. Science \& Justice. $1996 ; 36(1)$ : 41-5.

10. Dorough H.W., Torstenson J.H. Analysis of carbamate insecticides and metabolites. Chromatogr. Sci. $1975 ; 13$ : 212-23.

11. Moriya F., Hashimoto Y. Comparative studies on tissue distributions of organophosphorus, carbamate and organochlorine pesticides in decedents intoxicated with these chemicals. J. Forensic. Sci. 1999 ; 44(6) : 1131-5.

12. Tsatakis M. More fatal methomyl poisonings in Crete. Science \& Justice 1998 ; 38(4) : 279-83.

13. Drummer O.H. Stability of drugs post-mortem: A review. In: The International Association of Forensic Toxicologists XXXV Annual meeting, Padova, Italy, 2428 août 1997. Ce document peut être consulté sur le site : http://www.tiaft.org/tiaft97/index.html 